\title{
Germanica
}

\section{Mimesis, Moderne, Mythos: Dea Lohers Manhattan Medea}

Conte, mythe, morale: Manhattan Medea de Dea Loher

Fairytale, myth and morality: Dea Lohers Manhattan Medea

\section{Marcela Požárek}

\section{OpenEdition}

\section{Journals}

Édition électronique

URL : http://journals.openedition.org/germanica/2554

DOI : 10.4000/germanica.2554

ISSN : 2107-0784

\section{Éditeur}

Université de Lille

\section{Édition imprimée}

Date de publication : 30 juin 2014

Pagination : 107-123

ISBN : 9782913857339

ISSN : 0984-2632

\section{Référence électronique}

Marcela Požárek, « Mimesis, Moderne, Mythos: Dea Lohers Manhattan Medea », Germanica [Online], 54 2014, Online erschienen am: 30 Juni 2017, abgerufen am 06 Oktober 2020. URL : http://

journals.openedition.org/germanica/2554; DOI : https://doi.org/10.4000/germanica.2554 


\title{
Mimesis, Moderne, Mythos: Dea Lohers Manhattan Medea
}

\author{
Marcela Požárek \\ Queen Mary Univertity of London, \\ Austrian and Comparative Litterature
}

Ausgehend von Dea Lohers Theaterstück Manhattan Medea sollen verschiedene Aspekte ihrer Poetik untersucht werden, die auf raffinierte Weise Versatzstücke aus Märchen und Mythen verwenden, um einen Blick auf moralische Wertkonzepte und dramaturgische Strategien dieser Dramatikerin zu werfen.

Dabei stellt sich vorrangig die Frage, inwieweit Lohers Interesse an antiken Stoffen zu einer Rückkehr von klassischen ,redramatisierten“ Themen führt, die in scharfem Kontrast zu Hans-Thies Lehmanns „dedramatisierten“ Bildern bei Breughel stehen und seinem Begriff von Parataxis ${ }^{1}$. Dabei wird die Handlung auf der Bühne nicht dramatisch zugespitzt, sondern soll wie ein tableau vivant mit gleichschwebender Aufmerksamkeit in seiner Bedeutung enthierarchisiert betrachtet werden. Anders Loher, die den klassischen Medeastoff als Matrize verwendet, um darauf ihr zeitgenössisches Sittendrama zu projizieren. Im Mittelpunkt der Untersuchung soll insbesondere Loher's raffinierter Umgang mit Verfremdungen klassischer Figuren stehen, die einerseits

1. - Bernd Stegemann: Lektionen 1 Dramaturgie, Verlag Theater der Zeit, Berlin 2009. S.296. Quelle 52, Hans-Thies Lehmann: Postdramatisches Theater. „Diese Stillstellung ist eng verbunden mit dem narrativen Charakter der Bilder. Sie sind in auffallender Weise dedramatisiert: jeder Einzelheit scheint das gleiche Gewicht zuzukommen, die für die dramatische Darstellung typische Zuspitzung und Zentrierung mit der Trennung von Haupt- und Nebensache.“ 
eine Rückkehr von traditionellen dramatischen Dramenstoffen suggerieren, diese aber gleichzeitig durch Mittel der Ironisierung brechen und in Frage stellen. Die Figur der Medea war dabei schon immer theaterästhetisch von grosser Wirksamkeit, ist sie doch von starker Emotionalität geprägt: Jede Emotion hat ihre Gesten, ihren Blick, eine eigene Haltung, eine bestimmte Form.

In der Tragödie des Euripides erscheint Medea von Anfang an in einem Zustand extremer Passion, bald körperlich erstarrt und reglos wie Dürers Melencolia, bald schreiend, fürchterlich durch die hohen Säle heulend. Lange bevor man sie sieht, hört man diese Schreie, diese Flüche aus dem Innern des Palastes. Sie ist besessen von Visionen des Tötens, von Rache an allem, was mit Jason nur irgendwie zu tun hat: Rache an seiner neuen Braut, Rache an deren Vater, Rache an Jason selbst. Diese Medea, wie sie im Auftakt der Tragödie erscheint, ist eine der unerhörtesten Studien von Weiblichkeit, die es je gegeben hat und je geben wird. Sie ist unbedingt im Lieben wie im Hassen. Ihre ganze Person wird Gefühl, wird Liebe und Hass zugleich².

Wie diese bedingungslose Leidenschaft in ihrer Konsequenz dargestellt wird, ohne in klischeehaftes Pathos abzugleiten, ist ein komplexer Balanceakt, der Lohers Medea-Stück in einem gesellschaftspolitisch und produktionsästhetisch höchst ambivalenten Licht erscheinen lässt. Gleich zu Beginn von Manhattan Medea, als Auftragsarbeit für den steirischen herbst entstanden, stellt sich die Frage nach Form und Funktion der Antike und deren moderner Transformation, bei einem Theaterstoff, der von Korinth nach New York transponiert, einiges an kulturhistorischen Allusionen aufwirft. Während bei Euripides noch steht: „Der Schauplatz ist der Vorhof des Palastes Medeas in Korinth"3, heisst es bei Loher lapidar: „Manhattan, Gegenwart. Ein reiches Haus auf der 5th Avenue"4. Die Grossstadt wird hier zur Ikone des Kapitalismus, oder wie es Heiner Müller treffend definierte:

Die sieben Hauptzüge des Modernismus bzw. ihre postmodernistische Variation in dem von Ihab Hassan formulierten Steckbrief beschreiben New York so gut wie den Orpheus-Mythos in der Version Ovids oder Becketts Prosa. Eine Stadt, die sich aus ihrem Zufall konstituiert. Ein Gebilde, das aus einer eigenen Explosion besteht. Die Metropole des Dilettantismus: Kunst ist was man will, nicht was man kann. Eine

2. - Peter von Matt, Die Intrige, Theorie und Praxis der Hinterlist. XVI Medea. Hanser Verlag, München 2006, S.145.

3. - Euripides: Medea, Reclam Verlag, Stuttgart 1996, S. 3.

4. - Dea Loher, Manhattan Medea, Blaubart - Hoffnung der Frauen, Verlag der Autoren, Frankfurt a.M.1999, S. 8-9. 
elisabethanische Stadt: der Anschein von Wahl ist ein Vorschein von Freiheit ${ }^{5}$.

Der Charakter des Zufälligen, Provisorischen und Prekären dieser Stadt wird auch von Loher aufgegriffen, zudem wird das Figurenarsenal bei ihr reduziert und in moderner Weise karikiert. Was in der Antike ein Bote darstellt, übernimmt bei Loher „Deaf Daisy“, ein tauber Transvestit. Medea selbst bezeichnet sich als „eine Diebin, Dealerin. Vielleicht nur Hure, harmlos.“ Medea als ein modernes Stück über Außenseiter, die Hauptprotagonisten Medea und Jason sind in Amerika gestrandete Emigranten, deren schäbige Existenz sich in runtergekommenen Stundenhotels abspielt, ohne Aussicht auf Aufstieg. Jason, liiert mit Claire, einer Studentin der Juilliard School aus reichem Haus transformiert sich vom Emigranten zum Emporkömmling mit Ambitionen in der New Yorker High Society, sein künftiger Schwiegervater ist bei Loher ein zu schnellem Geld gekommener „Sweatshop-Boss“ und sitzt im Rollstuhl. Die antike Figur der Medea ist von dieser einfachen Charakteristik im Grunde nicht weit entfernt, wie Peter von Matt lapidar bemerkt:

Sie ist eine Magierin und Zauberin, Hexe großen Stils, [...]. Durch die Liebe zu Jason lebt Medea griechisch unter Griechen, bleibt aber doch auch eine Fremde und außerhalb der Ehe einsam [...]. Was für Jason damals ein aufregendes Abenteuer war, fernab am Schwarzen Meer, dieses exotische Hexenmädchen, wird ihm nun, zurück in Korinth, zur Last. Der Reiz ist weg. Er verliebt sich in die Tochter des Königs Kreon und ist im hohen Hause auch willkommen. Die neue Ehe ist beschlossene Sache. Nun fehlt nur noch Medeas Abschiebung6.

In der ersten Szene kommt Medea mit einem postmodernen Boten in Gestalt eines amerikanischen Türstehers in Berührung, der aber weitaus größere Ambitionen hegt, als den dekadenten Hausbewohnern und exquisiten Gästen Einlass zu gewähren, denn der Türsteher Velazquez malt in seiner Freizeit. Sein Name ist Programm und Zeichen für einen speziellen Diskurs, den Loher mit dieser Figur thematisiert.

Ich lerne durch Nachahmung. Noch ahme ich ihn nach, den anderen Velazquez. Noch unterwerfe ich mich jedem Detail in seinen Bildern in akribischem Studium, aber schon jetzt ist meine Nachahmung eine Neuerschaffung, die meinen Vorfahren bei genauerem Hinsehen übertrifft. Ich bin ein Meister der Kopie. Und doch - in dem ich sie signiere,

5. - Heiner Müller, Material, New York oder Das eiserne Gesicht der Freiheit, Reclam, Leipzig 1990, S. 98.

6. - Peter von Matt: Die Intrige, Theorie und Praxis der Hinterlist. XVI Medea. Hanser Verlag, München 2006, S. 145. 
mache ich ein Original aus ihr. Eine Kopie, die keine ist. Ein falscher Velazquez, der ein echter ist ${ }^{7}$.

Es geht hier also um Nachahmung, Mimesis, dem klassischen aristotelischen Thema par excellence, dessen man als Leser nicht sofort gewahr wird, da hier durch den künstlerisch ehrgeizigen Türsteher nicht auf die Antike, sondern auf spanische Hofmalerei verwiesen wird und somit auf adäquate Repräsentation von Macht, Hierarchie, Geschichte und herrschaftliche Insignien und selbstverständlich auch auf die Haltung des Künstlers zur Moral. Dea Lohers Medea lässt sich in Velazquez' Intention als äußerst gekonnte Nachahmung von Euripides lesen, die jedoch das Original letztlich übertreffen will, auch wenn es unweigerlich mit diesem durch den Vergleich für immer verbunden ist und nicht als autonomes Kunstwerk gelten kann. In Velazquez' Fall bedeutet dies konkret, dass er Medea in der 9. Szene mit den Worten: „Hier, das habe ich für Sie kopiert"8 einen Velazquez überreicht, der sich, in Flammen eines Feuers aufgehend, als ein Picasso entpuppt. Diese Anspielung auf klassische Malerei in Lohers Adaption erinnert an Thomas Bernhards Theaterstück „Ritter, Dene, Voss“, in dem das Porträtieren, die Nachahmung, als Ahnenkult und Porträtfimmel abgetan wird.
Natürlich sind die Bilder schlecht gemalt
Portraits sind immer schlecht gemalt
außer sie sind von Goya
aber Goya hat euch nicht gemalt
nie in Wien gemalt Goya ${ }^{9}$

Hier wird Goya, im Gegensatz zu Velazquez bei Loher, als der nicht zu übertreffende Künstler dargestellt. Die Aura des Originals bleibt in Bernhards Interpretation erhalten und unerreichbar, und jegliche Nachahmung wird als lächerliche Farce entlarvt. Das Mittel, um die Qualität des Originals zu übertreffen ist nicht Originalität, behauptet Lohers Velazquez, sondern äußerste Präzision in der Nachahmung, also technische Präzision.

Er will sich porträtieren lassen von mir. Aber er sagt, nicht seine Erscheinung, sondern die Wahrheit soll ich malen. Ich habe darüber

7. - Dea Loher : Manhattan Medea, Blaubart - Hoffnung der Frauen, Verlag der Autoren, Frankfurt a.M.1999, S.11.

8. - Dea Loher : Manhattan Medea, Blaubart - Hoffnung der Frauen, Verlag der Autoren, Frankfurt a.M.,1999, S. 60.

9. - Thomas Bernhard: Ritter, Dene, Voss. Programmheft Akademietheater Wien, Spielzeit 1986/87, S. 96. 
nachgedacht. Dann sage ich zu ihm, Mr Sawyer, Sir, sage ich, ich glaube, so weit bin ich noch nicht ${ }^{10}$.

Der Türsteher Velazquez wird hier dazu aufgefordert, hinter den Schein der Realität zu blicken, hinter die ästhetische Fassade auf die Wahrheit der Existenz, Kunst wird hier als Mittel der Erkenntnis eingesetzt, als Suche nach Authentizität. Diesen Anspruch hat auch die Autorin Dea Loher selbst. Lohers Dramentechnik und Benutzung von Mimesis wird von Birgit Haas als äußerst lobenswert begrüßt: „Aus dieser Perspektive sei darauf verwiesen, dass sich die dramatische Gegenwartsdramatik der Verantwortung gegenüber der Mimesis bereits stellt: Dea Loher verfolgt in ihren Stücken eine politisch engagierte Ästhetik, die sich an Schiller und Brecht abarbeitet; [...]"11. Haas sieht in der Mimesis geradezu eine Heilslehre gegen den postmodernen moralischen Verfall der zeitgenössischen Beliebigkeitsdramatik und eine Form von dramatischer Fertigkeit, die mit Erkenntnis einhergeht: „Um den postdramatischen Stillstand, der sich aus Adornos Zerschlagenheit der ästhetischen Parameter von Zeit, Raum und Subjekt ergab, hinter sich zu lassen, bietet sich eine altbewährte Methode an, die Mimesis. Mir ist dabei natürlich klar, dass hier nicht von der Nachahmung einer göttlichen Schöpfung die Rede sein kann. Vielmehr geht es um Mimesis im Sinne der Nachahmung einer dramatischen Ästhetik, die den Menschen als Subjekt im Gesamtgefüge seiner Beziehungen zu ihrem Mittelpunkt erwählt hat"12. Inwieweit es sich bei der Postdramatik um ein von Adorno verursachtes Phänomen des ästhetischen Stillstandes handelt, das durch Mittel der Mimesis überwunden werden kann, sei dahin gestellt. Interessant ist jedoch der Hinweis auf Mimesis als Nachahmung einer spezifischen dramatischen Ästhetik. Während der Türsteher Velazquez ein Loblieb auf traditionelle Maltechniken hält, zielt Haas'Argumentation auf eine Renaissance klassischer Tragödientechniken ab, wie sie bei zeitgenössischen deutschen Dramenautoren wieder an Beliebtheit zu gewinnen scheinen, wie auch Hans-Thies Lehmann in einer neuen Studie zum Schluss kommt:

So wird Tragödie wieder ein Vorstellungs- und Denkmodell jenseits der Träume der Aufklärung, jenseits der romantischen Verinnerlichung, jenseits des politischen Idealismus - eine Theaterform, in der radikale Skepsis, illusionslose Schilderung von Leid und Scheitern vorherrscht,

10. - Dea Loher : Manhattan Medea, Blaubart - Hoffnung der Frauen, Verlag der Autoren, Frankfurt a.M. 1999, S. 12.

11. - Birgit Haas: Plädoyer für ein dramatisches Theater, Passagen Verlag, Wien 2007. S. 48-49.

12. - Birgit Haas: Plädoyer für ein dramatisches Theater, Passagen Verlag, Wien 2007, S. 49. 
und die uns nicht durch Utopien oder Stilisierungen zum Heroischen bewegt, sondern durch das Mitgefühl mit menschlicher Schwäche, Gewahrwerden der Unzulänglichkeit. In diesem Sinne wirkt sie antiideologisch, und diese Qualität erweist sich mehr und mehr als ein nur allzu notweniges Antidotum zur alltäglichen platten Ideologisierung der Wirklichkeit, mit der das Bewusstsein gewöhnlich seine Ratlosigkeit überspielt ${ }^{13}$.

Was zeitgenössische Autoren und Regisseure an klassischen Tragödien also zu faszinieren scheint, ist die konsequente Darstellung menschlicher Realitäten, die in ihrer Radikalität nach schonungsloser Form verlangen.

Untersucht werden soll in diesem Zusammenhang, ob es Dea Loher in ihrer Medea Adaptierung gelingt, ,den Menschen als Subjekt im Gesamtgefüge seiner Beziehungen zu ihrem Mittelpunkt" darzustellen, oder ob ihr Versuch, den Stoff zu modernisieren, letztlich nicht zu einer Verflachung der Figuren und ihrem Wirkungskreis führt. Ausgehend von Gérard Genettes Definition: „Die Nachahmung ist zweifellos auch eine Transformation, stellt aber ein komplexeres Verfahren dar, da sie - um es wiederum sehr summarisch zu sagen - zunächst die Erstellung eines Modells der (sagen wir epischen) Gattungskompetenz erfordert, das, der Odyssee (und möglicherweise auch anderen Werken) als einzelnen Performanzen entnommen, zur Erzeugung einer unbeschränkten Zahl mimetischer Performanzen fähig ist" 14 muss bei der Bearbeitung eines antiken Dramenstoffes die Dynamik festgehalten werden, mit der tradierte Komponenten einer Handlung ausgelassen, verändert und verfremdet werden, und wie diese Transformationen die spezielle Qualität der Neufassung determiniert. So modern der Akt der Adaptierung des Stoffes erscheinen mag, so reaktionär kann eine neue Dramatisierung im Grunde sein. Es handelt sich dabei um nichts Anderes als um affirmative Kunst, um Form- und Machterhalt und Systemkonformität, die in scharfem Kontrast zur Interpretation der Antike beispielsweise eines Heiner Müller steht, wie er sie in folgendem Statement definiert:

Es ging um die Zuspitzung und um Pathos, das Treiben der Gedanken bis in die Konsequenz, bis zum Paradoxen. Ich halte das schon für notwenig. Ich glaube, was da drinsteckt, ist die Hoffnung. Wenn man die Sachen so weit treibt, sind sie besser einzuordnen in ein existie-

13. - Marion Tiedtke und Philipp Schulte (Hg.): Die Kunst der Bühne, Positionen des zeitgenössischen Theaters. Hans-Thies Lehmann: «Michael Thalheimer, Gestenchoreograph», Theater der Zeit, Berlin 2011, S. 86.

14. - Gérard Genette: Palimpsest, Die Literatur auf zweiter Stufe, Suhrkamp, Frankfurt a.M., 1993, 9-18, S. 16. 
rendes Wert- oder Denksystem. Ich glaube, das ist die wesentliche Funktion von Kunst überhaupt, Wert- und Denksysteme in Frage zu stellen, sie unter Umständen auch zu sprengen. Ganz simpel formuliert: Die Funktion von Kunst ist es, die Wirklichkeit unmöglich zu machen 15 .

Der Türsteher ist alles andere als ein revolutionärer Realitätsabbilder, der künstlerische Beobachtung auf die Spitze treibt, ihm ist daran gelegen, sich der Wirklichkeit unterzuordnen, genau so, wie es letztlich auch Dea Lohers Stück Manhattan Medea tut. Wir haben mit dem Türsteher Velazquez eine komplexe Figur vor uns: ein unbedeutender Hobbymaler, der einen Bedeutenden nachahmt, eigentlich Türsteher ist und dabei Kopien produziert, die das Original übertreffen sollen, es aber nicht in Frage stellen werden.

Birgit Haas äußert sich in ihrer Studie ,Plädoyer für ein dramatisches Drama“ im Kapitel „Die Renaissance des dramatischen Dramas“ ausführlich und rühmlich zu Dea Lohers Werk und kommt dabei auch auf deren Realitätsabbildung zu sprechen: „Loher setzt sich nun in ihrem Werk dezidiert mit den Widersprüchen auseinander, die sich aus der subjektiven Wahrnehmung und ihrer objektiven Gestaltung ergeben"16. Im Falle ihres Medeatextes lässt sich jedoch behaupten, dass sich komplexe Wahrnehmungsmuster der Protagonisten in der objektiven Gestaltung der Dialoge als nivellierend darstellen. Schon zu Beginn des Stücks stellt die Hauptfigur Medea die Frage nach Mimesis und Wahrheit: „Ist das Gold echt. Der Marmor echt. Ist das echter Samt hinter den Fenstern, in dem das Licht sich fängt und der die Räume verhüllt vor fremden Blicken"17. Medea beobachtet Realität, die ihr als Trug erscheint, da sie sie nicht wirklich zu Gesicht bekommt. Deshalb ist ihre Wahrnehmung der Wirklichkeit ein Trugschluss. Sie betrachtet den herrschaftlichen Palast Kreons, bei Loher zum reichen Haus eines Emporkömmlings in der 5th Avenue umfunktioniert, und versucht hinter die Fassade zu blicken, den Ereignissen auf die Spur zu kommen. In diesem Zusammenhang ist entscheidend, dass es hierbei um Architektur, Interieur und Ästhetik geht, also in erster Linie auch wieder, wie im Falle des Türstehers Velazquez, um Kunst und Schein. Der Raum, die Zimmer, in deren Machtbereich die Handlung stattfindet, werden von Samt verhüllt, das Geschehen im Innenraum bleibt unsichtbar, der außenstehende Beobachter ist auf Mutmaßungen ange-

15. - Heiner Müller: Gesammelte Irrtümer 2, Verlag der Autoren, Frankfurt a.M., 1990. S. 24.

16. - Birgit Haas: Plädoyer für ein dramatisches Theater, Passagen Verlag, Wien, 2007. S. 183.

17. - Dea Loher: Manhattan Medea, Blaubart - Hoffnung der Frauen, Verlag der Autoren, Frankfurt a. M., 1999, S. 10. 
wiesen. Medea bleibt nur im Vorzimmer der Ereignisse, in Lohers Bearbeitung steht sie buchstäblich auf der Straße, draußen vor der Tür und blickt auf Fenster.

La fenêtre propose un mode d'investigation du réel tout à fait singulier : non pas lecture mais déchiffrage, non pas déduction mais intuition. Objet symbolique, dans sa transparence virtuelle, elle invalide toute approche rationnelle et univoque, dénonce comme artificielle toute compartimentation étanche du réel. La fenêtre est à l'origine d'un processus de décryptage, le narrateur considérant la surface des choses comme une sorte de palimpseste dont il lui appartient de découvrir la signification ${ }^{18}$.

Es geht hier also grundsätzlich um Realitätsbetrachtung als Bildbetrachtung, um Bilder, die nicht genau betrachtet, sondern nur intuitiv dechiffriert werden können. Während der Türsteher Velazquez genau zu wissen scheint, was er sehen und malen will und es genauestens $\mathrm{zu}$ reproduzieren versteht, ist Medeas Beobachterposition eine unsichere. Nicht von ungefähr erwidert Medea dem Türsteher, als dieser sich beobachtet fühlt: „Ich betrachte nicht Sie“. Betrachtung allein reicht nicht aus, um die Geschehnisse begreifen zu können, sie müssen dechiffriert und in ein Zeichensystem überführt werden, das Sinn macht. Das Verhältnis zwischen Sichtbarkeit und Erkenntnisgewinn ist dabei zentral, wie schon Lessing festgehalten hat: „Dasjenige aber nur allein ist fruchtbar, was der Einbildungskraft freies Spiel lässt. Je mehr wir sehen, desto mehr müssen wir hinzu denken können. Je mehr wir dazu denken, desto mehr müssen wir zu sehen glauben"19. Die Suche nach Wahrheit wird erschwert durch die Tatsache, dass Medea nicht Einlass gewährt wird an den Ort, an dem Jason mit seiner neuen Geliebten weilt. Sie kann nur auf der Straße, in Lohers Fall die 5th Avenue, warten. Die Straße, das Prekäre an diesem Schwellenort, an dem nur gewartet und beobachtet werden kann, hat theatergeschichtliche Tradition:

L'un, utilisé surtout dans la comédie, consiste à représenter une rue, sur laquelle donnent les maisons des principaux personnages. On le trouve partout : dans Mélite de Corneille, dans le Dépit amoureux de Molière, dans les Plaideurs de Racine, etc... ; s'il s'agit d'une tragédie, la rue, trop peu noble, deviendra une place publique sur laquelle donneront palais ou temples ; tel sera le décor de Médée de Corneille ${ }^{20}$.

18. - Barbey d'Aurevilly, Les Diaboliques, Pascale Auraix-Jonchière, Éditions Gallimard, Paris, 1999, p. 102.

19. - Gotthold Ephraim Lessing: Laokoon, Reclam, Stuttgart 1990, S. 23.

20. - Jacques Scherer, La Dramaturgie classique en France, Librairie Nizet, Paris, 1973, p. 189. 
Da man nicht am Ort des Ereignisses steht, kann man nicht handeln, warten heißt hier also reden, Medea kann nur sprechen. Sie kann sprechend die Realität, in diesem Fall vor allem ihre Vergangenheit erfassen, nicht aber die Zukunft ändern, zu der sie keinen Zugang hat. Medea muss also im Vorzimmer der Ereignisse harren, und auch das hat seine symbolische Bedeutung, wie Roland Barthes im Zusammenhang mit Racineschen Tragödien erwähnt.

La Chambre est contiguë au second lieu tragique, qui est l'AntiChambre, espace éternel de toutes les sujétions, puisque c'est là qu'on attend. L'Anti-Chambre (la scène proprement dite) est un milieu de transmission ; elle participe à la fois de l'intérieur et de l'extérieur, du Pouvoir et de l'Événement, du caché et de l'étendu ; saisie entre le monde, lieu de l'action, et la Chambre, lieu du silence, l'Anti-Chambre est l'espace du langage : c'est là que l'homme tragique, perdu entre la lettre et le sens des choses, parle ses raisons ${ }^{21}$.

Die Straße ist in Lohers Stück das Vorzimmer, der Durchgangsort, wo die Wahrheit nur als Gerücht erfasst und interpretiert wird und umso mehr die Phantasie regieren kann. Nachdem Medea erfährt, dass sich Jason mit einer jungen Dame des amerikanischen Establishments trifft, bemerkt sie: ,Zwei Liebesvögel - das würde ich gern mit eigenen Augen sehen"22. Im Zusammenhang mit Heiner Müllers Antikenbearbeitung spricht Genia Schulz vom ,zersetzten Blick“: „Die Metapher überführt das Sehen in Nichtsehen und Blindheit, lässt das Auge vornehmlich als bedecktes, geschlossenes, zerstörtes, getäuschtes in Erscheinung treten"23. In Lohers Stück handelt es sich um die konkrete Metapher des Bildes, des paradoxerweise unsichtbaren Bildes und des unsicheren Blicks.

Une contrainte esthétique devient ainsi moyen d'expression tragique. La volonté de style, qui fait du langage un discours poétique, élève en même temps toute mimique et toute la gesticulation au niveau du regard ${ }^{24}$.

Das narzisstische Wechselspiel zwischen ständig überhöhter Selbstkontrolle und einem voyeuristischen Beobachtungstrieb determiniert das Wahrnehmungsspektrum der Protagonistin Medea. In Euripides' Fassung lässt Medea den Blick nicht kontemplativ in die

21. - Roland Barthes, Sur Racine, Éditions du Seuil, Paris 1963, p. 9-10.

22. - Dea Loher, Manhattan Medea, Blaubart - Hoffnung der Frauen, Verlag der Autoren, Frankfurt a.M. 1999, S. 13.

23. - Heiner Müller, Material, Reclam Verlag, Leipzig 1989, S. 165.

24. - Hrsg. Wolfgang Thiele, Racine. Jean Starobinski, Racine et la poétique du regard, Wissenschaftliche Buchgesellschaft, Darmstadt, 1976, S. 201. 
Ferne schweifen, sondern sie bleibt bei sich, den Blick konzentriert von der qualvollen Realität abgewandt:

Das Auge nicht erhebend und vom Boden nie

Den Blick wegwendend; wie der Fels, wie wogende

Meerflut, vernimmt sie Freundestrost und Mahnungen 25 .

Ganz anders hingegen bei Loher: Das Schwergewicht auf den visuellen Reiz der Szenen ist eng gebunden an die anonyme Situation des herumstreunenden Blicks eines Voyeurs in der Masse der Metropolen, wie ihn Walter Benjamin in seiner Baudelaire-Studie charakterisiert: „Die Erscheinung, welche ihn fasziniert - weit entfernt, sich dem Erotiker in der Menge nur zu entziehen, wird ihm durch diese Menge erst zugetragen. Die Entzückung des Städters ist eine Liebe nicht sowohl auf den ersten als auf den letzten Blick“26. Medea bezeichnet sich selbst nicht als Flaneurin, sondern als „Diebin, Dealerin, Hure“27, wobei auch hier implizit die Straße als Tatort figuriert. Medeas Verhalten ähnelt dem eines Fotografen, eines Großstadtpaparazzis, der gekonnt auf der Lauer liegend, begierig die Realität in lauter Bildmotive stückelt und dadurch einen distanzierten Dialog mit dem Gegenüber spinnt, ohne diesem aber nahe zu kommen.

Das führt auf Seiten des handelnden Fotografen zu jener seltsamen Empfindung, zugleich Zeuge, Ankläger, Verteidiger und Richter zu sein, einer Empfindung des schlechten Gewissens, die sich in seinen Gesten widerspiegelt. Deshalb versucht er, sein Motiv in einem unbewachten Moment zu überraschen, um es in ein Objekt zu verwandeln. In Anbetracht dessen, dass Fotografieren ein Scheindialog ist, erschwindelt auch er das Motiv 28 .

Dieser Scheindialog mit dem Motiv, d.h. zwischen Medea und Jason, führt unweigerlich zu einem Gefühl, dass das Betrachtete, das, was sich vor unseren Augen auf der Bühne abspielt, nichts mit der Realität zu tun hat und eigentlich bereits eine Vorahnung des Todes in sich birgt. Jason betrachtet die Situation in diesem Zusammenhang nüchtern und pragmatisch:

Wir haben es nicht geschafft, Medea, miteinander.

Und es ist besser, wir sehen darauf

25. - Euripides: Medea, Reclam Verlag, Stuttgart, 1996, S. 5.

26. - Walter Benjamin: Charles Baudelaire, Ein Lyriker im Zeitalter des Hochkapitalismus, Suhrkamp Verlag, Frankfurt am Main, 1974, S. 43-44.

27. - Dea Loher: Manhattan Medea, Blaubart - Hoffnung der Frauen, Verlag der Autoren, Frankfurt a.M., 1999, S. 16.

28. - Vilém Flusser: Gesten, Fischer, Frankfurt am Main, 1994, S. 114. 
mit einem kühlen Blick und einem Herzen, das nicht zittert beim Hören der Wahrheit ${ }^{29}$.

Interessant ist hier das Bild des kühlen Blicks auf die Wahrheit, es suggeriert Distanznahme und ein emotionsloses Sezieren der Realität. Jasons sprachlich salopper Pragmatismus und die darauffolgenden antikisierend sentimentalen Worte lassen Lohers Medeapathos zwischen Lächerlichkeit und Kitsch oszillieren, statt in ihr eine radikale Skepsis aufzuzeigen. Moderne Alltagssprache wird hier wie eine leichtfertige Anbiederung an ein zeitgenössisches Publikum eingesetzt, um archaisierende Wortwahl in ihrer vermeintlichen Obskurität abzumildern. Obwohl die Form eingehalten, nachgeahmt und nur leicht abgewandelt wird, gibt es keinen eindeutigen künstlerischen Mehrwert im Stück zu verzeichnen. „Die Gegenwartsprobleme“ werden zwar zuschauerfreundlich gefällig aufbereitet, aber nicht wirklich dramaturgisch spannend reflektiert, wie es Birgit Haas in ihrer Studie behauptet:

Sie löst sich vom „Antiform-Zwang“, das heißt von den antimimetischen postmodernen Texten und sucht nach Mitteln, die die Gegenwartsprobleme entsprechend wiedergeben können. Ihr Interesse gilt nicht den Abstraktionen, sondern den Ereignissen und Situationen in ihrer Umgebung, für die die Geisteswissenschaft keine Erklärung parat hat ${ }^{30}$.

Medeas Haltung in der Auseinandersetzung mit Jason ist alles andere als kühl, sie betrachtet die Situation mit einem mortifizierenden Blick, sie inszeniert ihre eigene Bildrealität, Vilem Flusser schreibt in diesem Kontext:,,Vor allem die „Inszenierung“ einer Person vor dem KameraAuge des Fotografen wird derart zum Index der Vergänglichkeit“31. Der Schein des Wahrgenommenen trügt unweigerlich, hinter der Fassade lauert etwas, oder es gähnt eine gut verdeckte Leere und die Lüge: „Kannst Du ein Gefühl haben, das wahr ist. Lügner“ konstatiert Medea gegenüber Jason. Die starken Affekte, die flottierenden Emotionen werden durch die Distanz des examinierenden Blickes in Schach zu halten versucht, in ihrer Wirkung abgemildert und dadurch paradoxerweise genauer rezipiert ${ }^{32}$.

29. - Dea Loher : Manhattan Medea, Blaubart - Hoffnung der Frauen, Verlag der Autoren, Frankfurt a.M., 1999, S. 18.

30. - Birgit Haas: Plädoyer für ein dramatisches Theater, Passagen Verlag, Wien, 2007, S. 182-183.

31. - Ästhetik der Inszenierung: Dimensionen eines künstlerischen, kulturellen und gesellschaftlichen Phänomens. Hrsg. von Josef Früchtl und Jörg Zimmermann. Suhrkamp Verlag, Frankfurt am Main, 2001, S. 44.

32. - Manfred Smuda: Der Gegenstand in der bildenden Kunst und Literatur. Typologische Untersuchung zur Theorie des ästhetischen Gegenstands. Wilhelm Fink 
Loher positioniert in ihrem Theaterstuick Medea in eine imaginäre Mitte, dadurch entsteht der Eindruck einer hermetisch abgeschotteten Geschichte, die wie ein Kunstgriff wirkt, oder wie Friedrich Dürrenmatt in seiner Rede zur Verleihung des Schiller-Gedächtnispreises des Landes Baden-Württemberg im Jahre 1986 festhält:

...die falsche Weihe, die allzu große Mission, der tierische Ernst schadet auf der Bühne, die nicht die Welt sei, nicht einmal deren Abbild, sondern eine vom Menschen erdichtete, erfabulierte Welt, in der die Leiden und Leidenschaften gespielt seien und nicht erduldet werden müssten und in welcher der Tod selbst nicht etwas Schreckliches, sondern nur einen dramaturgischen Kniff darstelle ${ }^{33}$.

Der durchkalkulierte Racheplan braucht die richtige Bühne, den richtigen Ort, um als weibliches Drama der Verletzungen in seiner ganzen Intensität und Schärfe zur Geltung zu kommen, will doch die Rächerin der Außenwelt ihr inneres Inferno zeigen, dieses nach außen stülpen und ihre seelische Realität inszenieren.

La question de la vengeance reste irrémédiablement confuse si l'on ne distingue pas un registre du vindicatif et un registre du vindicatoire. Le désir vindicatif désigne toute l'énergie passionnelle de la colère, tendue vers l'abréaction plus ou moins violente d'un traumatisme ${ }^{34}$.

Jede Gefühlsregung verlangt vom Darsteller eine unverwechselbare Gestik, einen spezifischen Blick und eine eindeutige Haltung, eine Art Manual der Emotionen. Was der Maler auf die Leinwand bannt, den Ausdruck einer Emotion in eine bestimmte Geste, genau diese Gefühlsregungen verfolgt der Zuschauer mit Spannung auf der Bühne. Wie schwierig es ist, den Medea-Stoff für ein zeitgenössisches Publikum auf intelligente Weise zu inszenieren, zeigt eine Rezension einer Inszenierung von Jacques Lassalle im Teatro Romano di Verona, in der Euripides' Medea von Isabelle Huppert dargestellt wird.

[...] an symbolischen Elementen sind zu erwähnen: der Ort und die Zeit, der Strand (von Korinth) und das Wasser, die Stadt und das Haus (nur evoziert). Die Tatsachen bestätigen eine der Wahrheiten zeitgenössischen Theaters: die Unfähigkeit von der Realität zu abstrahieren, von etwaigen Analogien zwischen der Vergangenheit (dem Fremden) und

\footnotetext{
Verlag, München, 1979, S. 69: „Ob es sich nun um Panoramen, Milieuschilderungen, Interieurs oder Personen in ihrem Aussehen handelt, immer ist die Darstellung durch den ,Blick' eines Beobachters motiviert und legt eine quasi-optische Konkretisation nahe.“

33. - Friedrich Dürrenmatt, Versuche, Diogenes Verlag, Zürich 1991, S. 97.

34. - La Vengeance, Études d'ethnologie, d'histoire et de philosophie, volume 4 : La Vengeance dans la pensée occidentale, textes réunis et présentés par Gérard Courtois, Éditions Cujas, Paris 1967, p. 55.
} 
der Aktualität (dem Alltäglichen). Sowohl Lassalle, als auch Huppert haben wiederholt in Interviews auf die Boulevardpresse, Justizfälle und darauffolgende Prozesse hingewiesen, sie haben, wenn nicht Medea und ihr Verbrechen, so doch deren Wiederholung zu rechtfertigen versucht: gibt es nicht auch heute Mütter, die ihre Kinder umbringen? Bei solchen Rechtfertigungen, aus denen nur eine reduzierte Aufführung hervorgehen kann, und deren Existenzialismus nur zwischen Pathologie (die Schreie Medeas im Haus) und einem gewissen Rationalismus oszilliert, lässt sich die Spur der heute dominierenden Moral verfolgen: Warum eigentlich nicht? Warum könnte man es nicht tun ? $^{35}$

In dieser Rezension wird sehr genau die Gratwanderung geschildert, die Theaterregisseure mit Adaptierungen antiker Dramenstoffe bestreiten müssen. Die Spannung, die zwischen antiken Konflikten und zeitgenössischer Realität entsteht, kann entweder produktiv als Radikalität im Sinne Heiner Müllers fruchtbar gemacht, oder aber nur als eine melodramatische Kulisse verwendet werden. Medea ist dabei keine Figur, deren Schicksal von einfachen, klaren Emotionen determiniert wird, ihr extremes Register an Gefühlen kommt auf der Bühne deshalb so gut zur Geltung, weil Emotionen ihre Existenz sind.

Bei der Auseinandersetzung mit Gefühlen im Theater rücken die Schauspieler bzw. das Wechselverhältnis von Bühne und Publikum in den Blickpunkt. Über Jahrhunderte wurde diese Beziehung ästhetisch, theoretisch und methodisch unterschiedlich beschrieben und gewichtet. Für die Konzeptualisierung von Emotionalität im Theater ist der Rekurs auf die Antike allerdings ein wichtiger Bezugspunkt ${ }^{36}$.

Medea ist Gefangene ihrer Erinnerungen, denen sie paradoxerweise ihre Existenz, die Existenz ihrer Geschichte verdankt. Sie verfällt ihren Leidenschaften wie einer Krankheit, sie versinkt im Hass. Durch qualvolle Selbstanalyse gelangt sie zur Einsicht, dass das Maß der Emotion

35. - Corriera della sera: «La Huppert, una Meda poco eroica», 6.7.2000, S. 48. Deutsche Übersetzung M.P.

... vale a dire da elementi simbolici: il luogo e il tempo, la spiaggia (di Corinto) e l'acqua, la città e la casa (solo evocati). Nei fatti, si conferma una delle verità del teatro contemporaneo: la sua impotenza a prescindere dal presente, da eventuali analogie tra il passato (l'estraneo) e l'attuale (ciò che sapiamo, o crediamo di sapere), fra il mistero (di un testo) e l'oscuro (della vita quotidiana). Sia Lassalle che la Huppert hanno a piu riprese citato nelle interviste la cronaca nera, i casi giudiziari e i processi che ne segueno; hanno entrambi tentato di giustificare, se non Medea e il suo crimine, la ricorrenza di esso: non vi sono anche oggi madri che uccidoni i loro figli? In tale giustificazione, da cui discende uno spettacolo fatalmente riduttivo, e il cui esistenzialismo oscilla tra la patologia (le urla die Medea da dentro casa) e un certo razionalismo, scorgiamo la traccia della morale oggi dominante: perche no? Perche, dopotutto, non si potrebbe fare?"

36. - Martin Harbsmeier, Sebastian Möckel, Hrsg. : Pathos, Affekt, Emotion. Transformationen der Antike, Suhrkamp, Frankfurt, 2009, S. 320. 
größer ist, als sie verkraften kann. Daraus entsteht das Monströse der großen Frauen der antiken Dramengeschichte, sie gehen am Übermaß ihres Gefühls unter: Medea, Elektra, Antigone oder Phädra. Peter von Matt charakterisiert diesen Typus wie folgt: „Die Frau, die aus blankem Hass besteht, es weiß und will und gut findet. Ihr Umriss ist monströs, soll monströs sein. Er zeigt, was das Monstrum per se ist: die schreckliche Ausnahme. [...] So tritt Hofmannsthals Elektra auf die deutsche Bühne.“37

Aus den Aufzeichnungen des Wiener Musikwissenschaftlers Eduard Hanslick erfahren wir sehr viel Aufschlussreiches über Theaterinszenierung im Paris des 19. Jahrhunderts, über den allgemein herrschenden Zuschauergeschmack und über eine bestimmte Theatertradition, die deutsche und französische Ästhetiken in diesem Bereich unterscheiden und die bis in die zeitgenössische Dramenrezeption ihre Gültigkeit hat und sich insbesondere in Lohers Medea-Adaption widerspiegelt. Es geht um die adäquate Darstellung von Pathos und Emotion auf der Bühne, einem Thema, dass französische Theaterleute laut Hansick ganz anders angehen als in Deutschland. So lange das ,Französische' für einen gewissen frivolen Stil hinhalten kann, ist alles bestens, was Hanslick aber weniger goutiert, ist die Tragödientradition der französischen Klassik, wie ein Zitat übers „théâtre français“ in seiner Autobiographie „Aus meinem Leben“ illustriert:

Auch Tragödien von Racine und Voltaire sah ich auf dem Théâtre français wieder zum Leben erweckt. In meinen Augen allerdings ein trauriges Leben. Ich fand die Dichtungen konventionell, unnatürlich, ungenießbar; die Aufführungen desgleichen. Den „Britannicus“ von Racine vermochte ich nicht auszuhalten. So widerwärtig hohl und prahlerisch berührten mich Spiel und Sprache dieser tremolierenden Deklamatoren. Als aber unmittelbar auf die Tragödie ein Scribesches Lustspiel folgte, bewunderte ich die Franzosen als die ersten Schauspieler der Welt ${ }^{38}$.

Bemerkenswert ist der hier geschilderte Kontrast zwischen dem Pathos der Racineschen Tragödie und der darauffolgenden Frivoliät des Scribeschen Lustspiels. Racines Dramen stehen im Zeichen tragischer Weltanschauung, die sich in den passionierten Gefühlsexzessen seiner Protagonisten manifestiert, genauso verhält es sich im Grunde genommen mit dem Medeastoff. Die starken Emotionen finden Halt in kühner Rhetorik. „Die Stücke lassen sich als selten unterbrochene

37. - Peter von Matt, Die Intrige, Theorie und Praxis der Hinterlist. XVI Medea. Hanser Verlag, München, 2006, S. 388.

38. - Eduard Hanslick, Aus meinem Leben, London, Gregg International Publishers, London 1971, S. 87. 
Wortgefechte charakterisieren. Das persuasive Element ist dabei von höchster Bedeutung"39. Hanslicks harsches Urteil über das französische Theaterrepertoire des Klassizismus passt sehr gut in einen Diskurs, der über die Vorzüge und Stärken französischer Dramen seit Jahrzehnten geführt wird. So äußerte sich Germaine de Staël sehr detailliert über die Unterschiede der deutschen und französischen Theatertradition und kommt dabei zu folgendem Schluss:

Wie mir scheint, kann niemand leugnen, dass die Franzosen in der Kombination der Theatereffekte am geschicktesten sind; auch in der Feierlichkeit und Würde der Situationen und des tragischen Stils behaupten sie den Vorrang vor allen anderen Nationen. Merkwürdig ist, dass es von diesen beiden Nationen gerade die Franzosen sind, die getragenste Würde im tragischen Stil verlangen. Aber gerade weil die Franzosen für den Schmerz empfänglicher sind, wollen sie hier keinen Anlass dazu geben, während nichts den unerschütterlichen Ernst der Deutschen stören kann ${ }^{40}$.

Der rasende Diskurs Medeas suggeriert die Möglichkeit, das Leben, trotz seiner absolut chaotischen Zusammenhänge im Griff behalten zu können. Dieser Diskurs ist durch eine dramatische Rhetorik gekennzeichnet, gleichzeitig kämpft die Figur um ihre emotionale Stabilität. Das radikale Wegbrechen der Identität, die Auflösung, Zerstörung macht sich in einer sprachlichen Verknappung bemerkbar, die Worte versanden, alles verstummt. Diesem Sich Abwenden von der Welt, diesem haltlosen Blick auf das Nichts geht ein massiver Überdruss an Weltlichkeit voraus. Die Abscheulichkeiten der Welt spiegeln sich für Medea in den Menschen wieder, die die Masken der Anständigkeit tragen. Die konsequente Vernichtungsarbeit, die sich in Medeas Drama abspielt, hat paradoxerweise zur Folge, dass alles was eigentlich verschwinden sollte, umso mehr präsent ist und durch das insistierende Hinweisen sogar mehr zum Vorschein kommt. Die letzten Worte Medeas in Lohers Stück sind denn auch bezeichnenderweise folgende:

Von jetzt an

werde ich

eine lebend Tote $\operatorname{sein}^{41}$.

39. - Hrsg. Wolfgang Thiele, Racine. Peter France: Funktional-persuasive Rhetorik,Wissenschaftliche Buchgesellschaft, Darmstadt, 1976, S. 294-295.

40. - Germaine de Staël, Über Deutschland. Über die dramatische Kunst. Reclam, Stuttgart, 1962, S. 194.

41. - Dea Loher, Manhattan Medea, Blaubart - Hoffnung der Frauen, Verlag der Autoren, Frankfurt a. M., 1999, S. 61. 
An diesem Beispiel lässt sich in exemplarischer Weise zeigen, dass Handlung nicht etwa mit äußeren Taten verwechselt werden darf, sondern auch Gefühlsabläufe und Gemütszustände nota bene in ihrer dramatischen Ausprägung, als Handlungen gelten können, oder wie es der Theatertheoretiker Bernhard Asmut definiert:

Im Sinne Lessings bezeichnet man die Gedanken und Gefühle seit dem 18. Jahrhundert gern als innere Handlung. Damit ist allerdings weniger ein einzelner psychischer Vorgang gemeint als die zusammenhängende Entwicklung aller derartiger Vorgänge in einem Drama. Der summative Begriff „Handlung“ (mythos), oft auf die „äußere Handlung“ beschränkt, wird so ergänzt bzw. differenziert ${ }^{42}$.

So brutal Medeas Geschichte, die über Jahrhunderte ihre Wirkung auf der Bühne nicht verfehlt, so stark ist dieser Stoff davon geprägt, wie die Hauptfigur nicht nur agiert, sondern vor allem ihre eigene Lage reflektiert. Die dramatische Spannung baut sich hierbei also nicht so sehr im Handeln als im Denken auf. Der absolute Anspruch ihres Denkens wird in Lohers Fassung durch tragische Worte erfasst:

Jetzt

ist kein Gesetz mehr

Ausser mir ${ }^{43}$

Dieser pathetische Absolutheitsanspruch wird in Lohers Stück immer wieder gebrochen durch die Beliebigkeit flapsiger Alltagssprache, die den Anschein des Natürlichen und Zeitgemäßen erwecken soll und dadurch starke Emotionen zwangsläufig abmildert. Letztlich stellt sich die Frage, welcher Preis für eine Nachahmung einer klassischen griechischen Tragödie, wie in diesem Fall Euripides' Medea, gezahlt werden muss. Welche Kompromisse geht Dea Loher ein, um den antiken Stoff in eine Gegenwart zu transportieren, denn: „Es geht um die mögliche Gegenwart und Zukunft der Tragödie. Sind Kategorien wie Schicksal, Heros, tragische Schuld, mythischer Starrsinn, Ausweglosigkeit, die wir mit Tragödie assoziieren, noch adäquat, unsere Wirklichkeit zu beschreiben?"44, wie Hans-Thies Lehmann konstatiert. Weil Loher aber Aspekte von tragischer Schuld und Ausweglosigkeit ihrem Drama nicht zumuten will, ist ihre Medea eine relativ banale Alltagsfigur, deren

42. - Bernhard Asmuth, Einführung in die Dramenanalyse, B. Metzlersche Verlagsbuchhandlung, Stuttgart, 1980, S. 6.

43. - Dea Loher : Manhattan Medea, Blaubart - Hoffnung der Frauen, Verlag der Autoren, Frankfurt a. M., 1999, S. 40.

44. - Marion Tiedtke und Philipp Schulte (Hg.), Die Kunst der Bühne, Positionen des zeitgenössischen Theaters. Hans-Thies Lehmann, «Michael Thalheimer, Gestenchoreograph», Theater der Zeit, Berlin 2011, S. 86. 
dramatisches Potenzial unausgeschöpft bleibt. Welche künstlerischen Möglichkeiten bei der Darstellung einer so komplexen Frauenfigur bestehen, hatte dabei schon Gotthold Ephraim Lessing beim Maler Timomachus scharfsinnig beschrieben.

Die Medea hatte er nicht in dem Augenblicke genommen, in welchem sie ihre Kinder wirklich ermordet; sondern einige Augenblicke zuvor, da die mütterliche Liebe noch mit der Eifersucht kämpfet. Wir sehen das Ende dieses Kampfes voraus. Wir zittern voraus, nur bald bloß die grausame Medea zu erblicken, und unsere Einbildungskraft gehet weit über alles hinweg, was uns der Maler in diesem schrecklichen Augenblicke zeigen könnte ${ }^{45}$. 
